\title{
Biologia reprodutiva de Macrobrachium olfersii (Wiegman) (Crustacea, Decapoda, Palaemonidae) coletados na Ilha de Santa Catarina, Brasil
}

\author{
Dib Ammar ${ }^{1}$ \\ Yara Maria Rauh Müller ${ }^{1}$ \\ Evelise Maria Nazari ${ }^{1}$
}

\begin{abstract}
Reproductive biology of Macrobrachium olfersii (Wiegman) (Crustacea, Decapoda, Palaemonidae) collected at Santa Catarina's Island, Brazil. The aim of this study is to characterize the reproductive features of Macrobrachium olfersii (Wiegman, 1836) from Santa Catarina's Island. The animals were collected in freshwater streams of the Ratones Hidrografic Basin (site A1) and at the Peri's Pond (site A2). In the laboratory, the procedures of sexual differentiation were carried out to classify the individuals in males, females or ovigerous females, followed by the determination of total weight $(\mathrm{g})$, quelipod relative weight $(\mathrm{g})$ and total length $(\mathrm{mm})$. The eggs were removed from the ovigerous females and counted. Sexual maturity and fecundity were analyzed based in the dimensions and number of eggs carried by ovigerous females. The water temperature on the capture sites varied from 17 to $29^{\circ} \mathrm{C}$ throughout the months of study. A total of 503 individuals were captured, with 129 individuals from site $\mathrm{A} 1$ and 374 from site A2. The mean total size and length was $49.65 \mathrm{~mm}$ and $3.79 \mathrm{~g}$ for the males, $38.75 \mathrm{~mm}$ and $1.61 \mathrm{~g}$ for the females and 36.19 $\mathrm{mm}$ and $1.08 \mathrm{~g}$ for the ovigerous females. At the sites $\mathrm{A} 1$ and $\mathrm{A} 2$, the individual sexual maturity was reached with 27.3 and $21.2 \mathrm{~mm}$ of total length, respectively, while populational sexual maturity was estimated for the 45.0 to $50.0 \mathrm{~mm}$ class at site A1 and for the 30.0 to $35.0 \mathrm{~mm}$ class at site $\mathrm{A} 2$. The ovigerous females carried an average of 3464 eggs at site A1, and 1440 eggs at site A2. These reproductive features, presented by $M$. olfersii, show the species' plasticity, which can be associated with environmental variations, contributing to its reproductive success.

KEY WORDS. Macrobrachium olfersii, fecundity, sexual maturity, environmental conditions
\end{abstract}

$\mathrm{Na}$ Ilha de Santa Catarina foram registrados representantes da família Palaemonidae (Rafinesque, 1815), identificadas como Macrobrachium acanthurus (Wiegmann, 1836), Macrobrachium carcinus (Linnaeus, 1758), Macrobrachium olfersii (Wiegmann, 1836), Macrobrachium potiuna (Müller, 1880), Palaemon pandaliformis (Stimpson, 1871) e Palaemonetes argentinus (Nobili, 1901) (MüLler et al. 1996, 1999).

Dentre estes camarões, $M$. olfersii é encontrado com freqüência em rios, riachos e canais nas diferentes regiões da Ilha de Santa Catarina, sendo a Lagoa do Peri e a bacia do rio Ratones, os locais com maior representatividade desta espécie (Müller \& Prazeres 1992; Müller et al. 1999).

1) Departamento de Biologia Celular, Embriologia e Genética, Universidade Federal de Santa Catarina. Caixa Postal 476, 88010-970 Florianópolis, Santa Catarina, Brasil. 
Macrobrachium olfersii é uma espécie predominantemente de água doce, que necessita de água salobra para completar seu ciclo de vida, principalmente para a reprodução e metamorfose larval (LiMA et al. 1997).

Esta espécie apresenta porte medianamente robusto (BOND-BUCKUP \& BUCKUP 1989), e assim como nos demais palemonídeos, machos e fềmeas apresentam portes bastante semelhantes, até ser atingida a maturidade sexual, quando iniciam-se os processos reprodutivos. As fêmeas passam então a apresentar um gasto energético consideravelmente superior, em virtude da produção dos ovos e organização da câmara incubadora, enquanto que os machos investem em crescimento somático tornando-se os maiores indivíduos da população (BoschI 1974; HARTNOLl 1982; ADIYODI \& SUBRAMONIAM 1983).

O número absoluto de ovos carregados em todas as desovas de uma fêmea é determinado por fatores genéticos e ambientais, sendo que o número relativo de ovos carregados em uma única desova, a fecundidade individual, exibe uma relação linear com o tamanho da fêmea (SHAKUnTALA 1977; VALENTI et al. 1989). De um modo geral, as espécies que produzem ovos volumosos têm uma fecundidade individual relativamente baixa, quando comparadas às que produzem ovos menores, que podem ser acomodados em maior número na câmara incubadora (PEREIRA \& GARCIA 1995).

Em muitos crustáceos, os processos reprodutivos estão na dependência, além do controle hormonal, das condições ambientais (LAUFER \& LANDAU 1991; QUACKENBUSH 1994; Fingerman 1995). De um modo geral, os palemonídeos tendem a apresentar ciclo reprodutivo anual, podendo ocorrer deslocamentos do período, bem como variações na duração das estações reprodutivas, devido principalmente às mudanças da temperatura (BOOLOOTIAN et al. 1959; MÜLLER \& PRAZERES 1992).

A família Palaemonidae é encontrada em número significativo em diferentes habitats, tanto em água doce quanto salobra, destacando-se por sua importância ecológica e econômica (COREY \& REID 1991). Assim, o conhecimento da biologia reprodutiva destes camarões é essencial para o desenvolvimento de programas de cultivo e de repovoamento de espécies nativas.

No presente trabalho pretende-se caracterizar indicadores reprodutivos da espécie nativa $M$. olfersii em duas localidades da Ilha de Santa Catarina, contribuindo assim para um melhor conhecimento da biologia deste camarão.

\section{MATERIAL E MÉTODOS}

Os animais foram capturados entre junho de 1994 e maio de 1996 em dois locais distintos da Ilha de Santa Catarina, através do arrasto de puçá malha $0,5 \mathrm{~cm}$ por entre as pedras e vegetação submersas. $\mathrm{O}$ esforço de captura foi padronizado em 10 minutos, sendo as coletas realizadas preferencialmente no período da tarde e registrados no local, a temperatura da água e o teor de salinidade. A área de estudo A1, localizada na Bacia Hidrográfica de Ratones ( $\left.27^{\circ} 28^{\prime} \mathrm{S}, 48^{\circ} 30^{\prime} \mathrm{W}\right)$ está situada na região noroeste, com um área total de $16 \mathrm{Km}^{2}$ (CECA/FNMA 1996). O local de captura apresenta águas cristalinas, fundo pedregoso, com profundidade máxima de $50 \mathrm{~cm}$, sendo que nas margens há pouca vegetação arbustiva e arbórea, conferindo muita 
luminosidade ao ambiente. A área de estudo A2, localizada no Parque Municipal da Lagoa do Peri $\left(27^{\circ} 45^{\prime} \mathrm{S}, 48^{\circ} 32^{\prime} \mathrm{W}\right)$, na região sul, está inserida em uma bacia hidrográfica de $20,3 \mathrm{Km}^{2}$, sendo $5,2 \mathrm{Km}^{2}$ a área da própria lagoa (CECA/FNMA 1996). O local de captura apresenta águas ligeiramente turvas, com fundo arenoso e farta vegetação aquática e aérea, o que confere ao ambiente pouca luminosidade.

Em laboratório, procedeu-se a diferenciação sexual dos indivíduos que apresentavam comprimento total superior a $20 \mathrm{~mm}$, através da análise das características sexuais secundárias como a presença do apêndice sexual masculino, a organização da câmara incubadora e a presença de ovos na câmara, sendo os exemplares diferenciados em machos, fêmeas e fêmeas ovígeras. Em seguida, os indivíduos foram fixados em formol $10 \%$ por 24 horas e conservados em álcool $70 \%$.

Foram registrados os valores de peso total $(\mathrm{g})$ e de peso relativo (g), este último determinado pelo peso do maior quelípodo presente nos machos. O comprimento total $(\mathrm{mm})$ foi obtido através da medida correspondente à distância entre as extremidades distais do rostro e do telso. A maturidade sexual individual foi determinada pelo comprimento apresentado pela menor fêmea ovígera capturada (MülLER et al. 1996), enquanto que a maturidade sexual populacional foi estimada para a classe de comprimento total em que se completa $50 \%$ do número de fêmeas da população estudada (PERRONE \& VIEIRA 1990). As fềmeas ovígeras tiveram seus ovos retirados da câmara incubadora e contados com registrador manual, para determinação da fecundidade individual. A fecundidade populacional foi estimada através da análise de regressão linear entre o comprimento total e o número de ovos. Os dados biométricos e de fecundidade foram agrupados por área de estudo (A 1, A2), sendo então submetidos ao teste-T e ANOVA one-way (Duncan), $p \geq 0,05$.

\section{RESULTADOS E DISCUSSÃO}

Durante o período de estudo a salinidade manteve-se em zero e a temperatura mostrou-se próxima nas áreas A1 e A2, variando de 17 a $29^{\circ} \mathrm{C}$, sendo possível observar um período de temperaturas mais elevadas, superiores a $25^{\circ} \mathrm{C}$, entre novembro e abril (Figs 1-2). Embora os registros da temperatura e da salinidade tenham apresentado valores muito semelhantes nas áreas estudadas, estas apresentam características ambientais distintas, que podem estar refletidas principalmente no tamanho populacional e nas variações de porte dos indivíduos. Contudo, os locais preferencialmente habitados por $M$. olfersii, nas áreas A1 e A2, correspondem aos ambientes descritos por MCNAMARA et al. (1985).

Nas áreas de estudo A1 e A2 foram capturados um total de 503 indivíduos, sendo 129 em A1 e 374 em A2, distribuidos conforme a tabela I.

Tabela I. Distribuição de machos, fêmeas e razão sexual correspondente de exemplares de M. olfersii capturados nas áreas de estudo A1 e A2, no período de junho de 1994 a maio de 1996.

\begin{tabular}{ccccc}
\hline Area de Estudo & Número de machos & Número de fêmeas & Número de fêmeas ovigeras & Razăo sexual \\
\hline A1 & 50 & 42 & 37 & 0,63 \\
A2 & 160 & 104 & 110 & 0,75 \\
\hline
\end{tabular}


A1

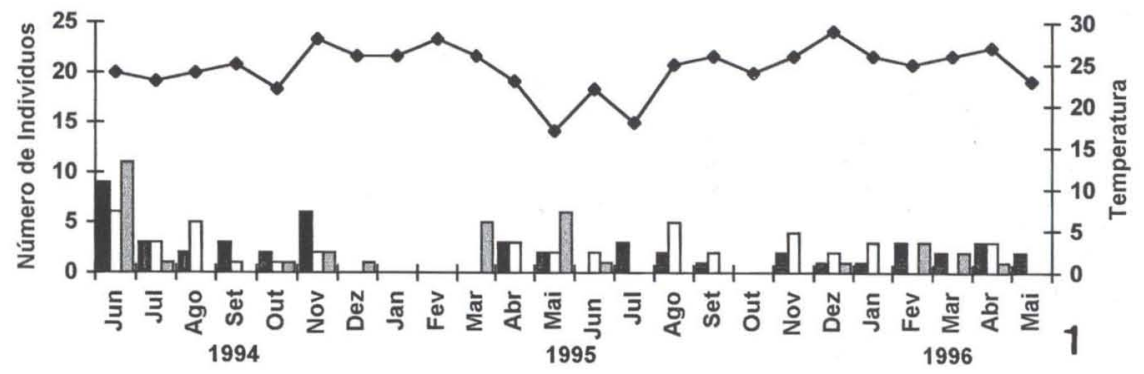

A2

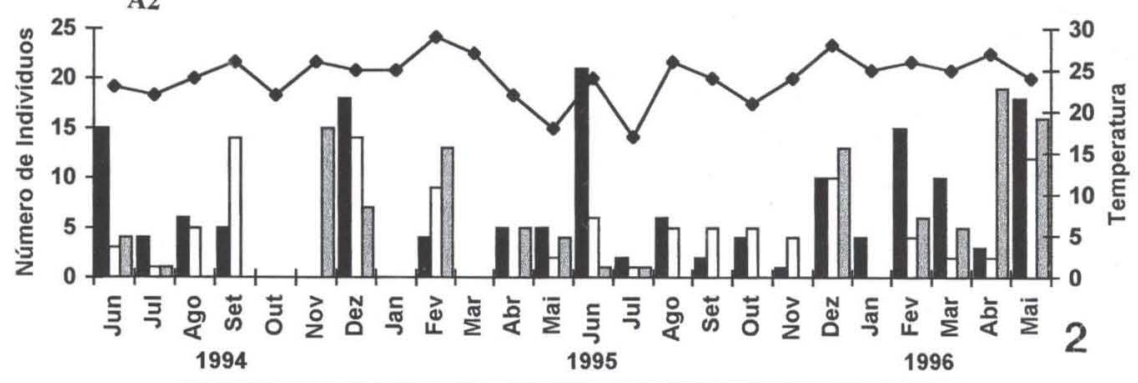

Machos $\square$ Fêmeas $\square$ Fêmeas Ovígeras $\multimap-$ Temperatura

Figs 1-2. Distribuição mensal da freqüência absoluta de $M$. olfersii nas áreas de estudo (1) $A 1$ e (2) A2 e flutuação da temperatura no período de junho de 1994 a maio de 1996.

Macrobrachium olfersii apresentou uma distribuição regular ao longo dos meses, já as fêmeas ovígeras apresentaram um período de presença bastante característico nas duas áreas. Na área de estudo Al (Fig. 1), as fêmeas ovígeras apresentaram uma ocorrência mais representativa no mês de junho de 1994, março e maio de 1995. Já na área A2 (Fig. 2), registrou-se uma maior representatividade de fêmeas ovígeras nos meses de novembro e dezembro de 1994, fevereiro e dezembro de 1995, abril e maio de 1996. Esta flutuação populacional parece ser indicativo de ciclo reprodutivo anual, caracterizado por períodos de presença e ausência de fêmeas ovígeras na população (GIESE \& PEARSE 1974). A presença de fêmeas ovígeras pode estar associada a períodos de elevação da temperatura, sendo a média registrada para estes meses igual a $25^{\circ} \mathrm{C}$. Segundo CARvalHo (1978), é observado períodos de ausência de fêmeas ovígeras, que correspondem aos tempo necessário à reestruturação do ovário e o começo de um novo ciclo reprodutivo.

O comprimento médio apresentado pelos machos estudados nas áreas Al e A2 foi de $49,65 \mathrm{~mm}$, já as fêmeas mediam $38,75 \mathrm{~mm}$, enquanto que as fềmeas ovígeras mediam $36,19 \mathrm{~mm}$. O peso total médio apresentado pelos machos foi de $3,79 \mathrm{~g}$, sendo que as fềmeas e fềmeas ovígeras pesavam respectivamente, $1,61 \mathrm{~g}$ e $1,08 \mathrm{~g}$. Os dados gerais referentes ao comprimento e peso de machos, fêmeas e fêmeas ovígeras nas áreas de estudo A1 e A2 estão apresentados na tabela II. O comprimento total organizado em classes (Tab. III), mostra que a maioria da 
população (93\%) está distribuída nas classes de comprimento superiores a $25,00 \mathrm{~mm}$ e inferiores a $60,00 \mathrm{~mm}$. Já para o peso (Tab. IV), $78 \%$ da população estudada encontra-se distribuída nas primeiras três classes.

Tabela II. Valores de comprimento total, peso e número de ovos de indivíduos de $M$. olfersii, coletados em A1 e A2, no periodo de junho de 1994 a maio de 1996. (DP) Desvio padrão, $(\bar{X})$ média.

\begin{tabular}{|c|c|c|c|c|c|c|c|c|c|c|c|c|}
\hline \multirow{2}{*}{\multicolumn{2}{|c|}{ Área de estudo }} & \multicolumn{3}{|c|}{ Comprimento total (mm) } & \multicolumn{4}{|c|}{ Peso $(g)$} & \multicolumn{4}{|c|}{ Número de ovos } \\
\hline & & Minimo Máximo & $\bar{x}$ & DP & Minimo & Máximo & $\bar{x}$ & DP & Minimo & Máximo & $\bar{x}$ & DP \\
\hline \multirow{3}{*}{ A1 } & Machos & $42,70-93,00^{*}$ & 59,75 & 10,92 & 2,35 & $-16,57$ & 5,86 & 3,71 & - & - & - & - \\
\hline & Fêmeas & $25,40-58,20$ & 45,80 & 9,83 & $0,18-$ & $-4,30$ & 2,51 & 1,28 & - & - & - & - \\
\hline & Fêmeas ovigeras & $27,30-60,00^{\star *}$ & 47,65 & 8,42 & $0,35-$ & $-4,42$ & 2,68 & 1,03 & 445 & 9052 & 3464 & 968 \\
\hline \multirow{3}{*}{$\mathrm{A} 2$} & Machos & $20,10-83,00^{*}$ & 45,50 & 13,75 & $0,06-$ & $-14,98$ & 3,12 & 3,46 & - & - & - & - \\
\hline & Fèmeas & $23,00-57,90$ & 36,30 & 7,87 & $0,15-$ & $-4,18$ & 1,30 & 1,08 & - & - & - & - \\
\hline & Fêmeas ovigeras & $21,20-59,30^{* *}$ & 35,10 & 10,25 & $0,25-$ & $-4,13$ & 1,00 & 0,74 & 410 & 4536 & 1440 & 833 \\
\hline & Machos & $20,10-93,00$ & 49,65 & 14,49 & $0,06-$ & $-16,57$ & 3,79 & 3,73 & - & - & - & - \\
\hline \multirow{2}{*}{\multicolumn{2}{|c|}{$\begin{array}{l}\text { GeralFêmeas } \\
\text { Fêmeas ovigeras }\end{array}$}} & $23,00-58,20$ & 38,75 & 9,35 & $0,15-$ & $-4,30$ & 1,61 & 1,24 & - & - & - & - \\
\hline & & $21,20-60,00$ & 36,19 & 8,57 & $0,25-$ & $-4,42$ & 1,08 & 1,01 & 410 & 9052 & 1557 & 1202 \\
\hline
\end{tabular}

${ }^{*}$ ) Indica diferenças significativas de comprimento entre A1 e A2 $(p \leq 0,005)$;

$\left({ }^{* *}\right)$ indica diferenças significativas de comprimento entre A1 e A2 $(p \leq 0,005)$

Tabela III. Distribuição em classes de comprimento total $(\mathrm{mm})$ dos exemplares de M. olfersii coletados em Bacia Hidrográfica de Ratones A1 e Parque da Lagoa do Peri A2, no período de junho de 1994 a maio de 1996.

\begin{tabular}{|c|c|c|c|c|c|c|}
\hline \multirow{2}{*}{$\begin{array}{l}\text { Classe de comprimento } \\
\qquad(\mathrm{mm})\end{array}$} & \multicolumn{3}{|c|}{ A1 } & \multicolumn{3}{|c|}{ A2 } \\
\hline & Machos & Fèmeas & Fêmeas ovigeras & Machos & Fêmeas & Fêmeas ovigeras \\
\hline $20-25$ & - & - & - & 4 & 6 & 5 \\
\hline $25-30$ & 1 & 2 & 1 & 15 & 27 & 16 \\
\hline $30-35$ & 2 & 3 & 3 & 26 & 26 & 38 \\
\hline $35-40$ & 2 & 8 & 2 & 28 & 23 & 32 \\
\hline $40-45$ & 3 & 6 & 5 & 21 & 13 & 15 \\
\hline $45-50$ & 4 & 9 & 8 & 19 & 8 & 2 \\
\hline $50-55$ & 10 & 11 & 10 & 19 & 3 & 2 \\
\hline $55-60$ & 11 & 3 & 7 & 9 & - & 1 \\
\hline $60-65$ & 9 & - & 1 & 12 & - & - \\
\hline $65-70$ & 4 & - & - & 1 & - & - \\
\hline $70-75$ & 2 & - & - & 1 & - & - \\
\hline $75-80$ & 1 & - & - & 1 & - & - \\
\hline $80-85$ & - & - & - & 1 & - & - \\
\hline $85-90$ & - & - & - & - & - & - \\
\hline $90-95$ & 1 & - & - & - & - & - \\
\hline
\end{tabular}

Quanto aos machos que apresentavam peso total superior a $3 \mathrm{~g}$, obteve-se peso total médio de $6,39 \mathrm{~g}$, sendo deste total $2,55 \mathrm{~g}$ o peso médio do maior quelípodo, o que corresponde a $40 \%$ do peso corporal total, relação esta não observada para as fêmeas. Este crescimento diferenciado entre as partes do corpo, a partir de uma determinada idade, caracteriza um dimorfismo sexual acentuado, onde o quelípodo desempenha função reprodutiva, além de diferenciar morfologicamente machos de fêmeas. 
Tabela IV. Distribuição em classes de peso (g) dos exemplares de $M$. olfersii coletados na Bacia Hidrográfica de Ratones (A1) e Parque da Lagoa do Peri (A2), no periodo de junho de 1994 a maio de 1996.

\begin{tabular}{|c|c|c|c|c|c|c|}
\hline \multirow{2}{*}{$\begin{array}{l}\text { Classe de comprimento } \\
\qquad(\mathrm{mm})\end{array}$} & \multicolumn{3}{|c|}{ A1 } & \multicolumn{3}{|c|}{ A2 } \\
\hline & Machos & Fêmeas & Fèmeas ovigeras & Machos & Fêmeas & Fêmeas ovigeras \\
\hline $0,01-1,0$ & 4 & 10 & 4 & 65 & 77 & 70 \\
\hline $1,0-2,0$ & 6 & 10 & 5 & 24 & 13 & 31 \\
\hline $2,0-3,0$ & 9 & 13 & 15 & 24 & 6 & 7 \\
\hline $3,0-4,0$ & 5 & 7 & 9 & 11 & 7 & 1 \\
\hline $4,0-5,0$ & 6 & 2 & 4 & 13 & 1 & 1 \\
\hline $5,0-6,0$ & 4 & - & - & 8 & - & - \\
\hline $6,0-7,0$ & 4 & - & - & 5 & - & - \\
\hline $7,0-8,0$ & 5 & - & - & 2 & - & - \\
\hline $8,0-9,0$ & 2 & - & - & - & - & - \\
\hline $9,0-10,0$ & 1 & - & - & - & - & - \\
\hline $10,0-11,0$ & 1 & - & - & 3 & - & - \\
\hline $11,0-12,0$ & 1 & - & - & 2 & - & - \\
\hline $12,0-13,0$ & - & - & - & - & - & - \\
\hline $13,0-14,0$ & 1 & - & - & 2 & - & - \\
\hline $14,0-15,0$ & - & - & - & 1 & - & - \\
\hline $15,0-16,0$ & - & - & - & - & - & - \\
\hline $16,0-17,0$ & 1 & - & - & - & - & - \\
\hline
\end{tabular}

Os valores de comprimento e peso registrados para $M$. olfersii confirmam que este é um camarão de médio porte (BOND-BUCKUP \& BUCKUP 1989) e que os machos são os maiores indivíduos da população, como reporta DUMBAULD et al. (1996) para outras espécies de camarões.

Em M. olfersii, assim como em M. carcinus (Grazziani et al. 1993), a formação de um quelípodo robusto nos machos, é necessária para o estabelecimento de territórios, bem como defender a fêmea durante o comportamento de corte, quando se dá a muda pré-copulatória.

As variações de peso e comprimento apresentadas por $M$. olfersii em localidades distintas, indicam a plasticidade destes camarões em adaptar-se às diferentes condições ambientais, mantendo porém as características da espécie quanto ao porte apresentado.

As fêmeas de $M$. olfersii estudadas em A1 e A2 atingem a maturidade sexual individual com 27,3 e 21,2mm de comprimento total, respectivamente. A maturidade sexual populacional foi estimada para a classe de comprimento entre 45,0 a $50,0 \mathrm{~mm}$ em A1 e 30,0 a 35,0mm em A2 (Tab. III).

O número de ovos registrado para as fêmeas ovígeras em Al variou de 445 a 9052 ovos, apresentando uma fecundidade individual média de 3464 ovos. Em A2, o número de ovos variou de 410 a 4536 ovos, sendo a fecundidade individual média de 1440 ovos (Tab. II).

A fecundidade populacional estimada para $M$. olfersii na Ilha de Santa Catarina está representada nas equações de fecundidade (F), obtidas para as fềmeas que apresentavam comprimento total entre 27,30 e $60,00 \mathrm{~mm}$ para Al e 21,20 e $59,30 \mathrm{~mm}$ para A2 (Figs 3-4). 

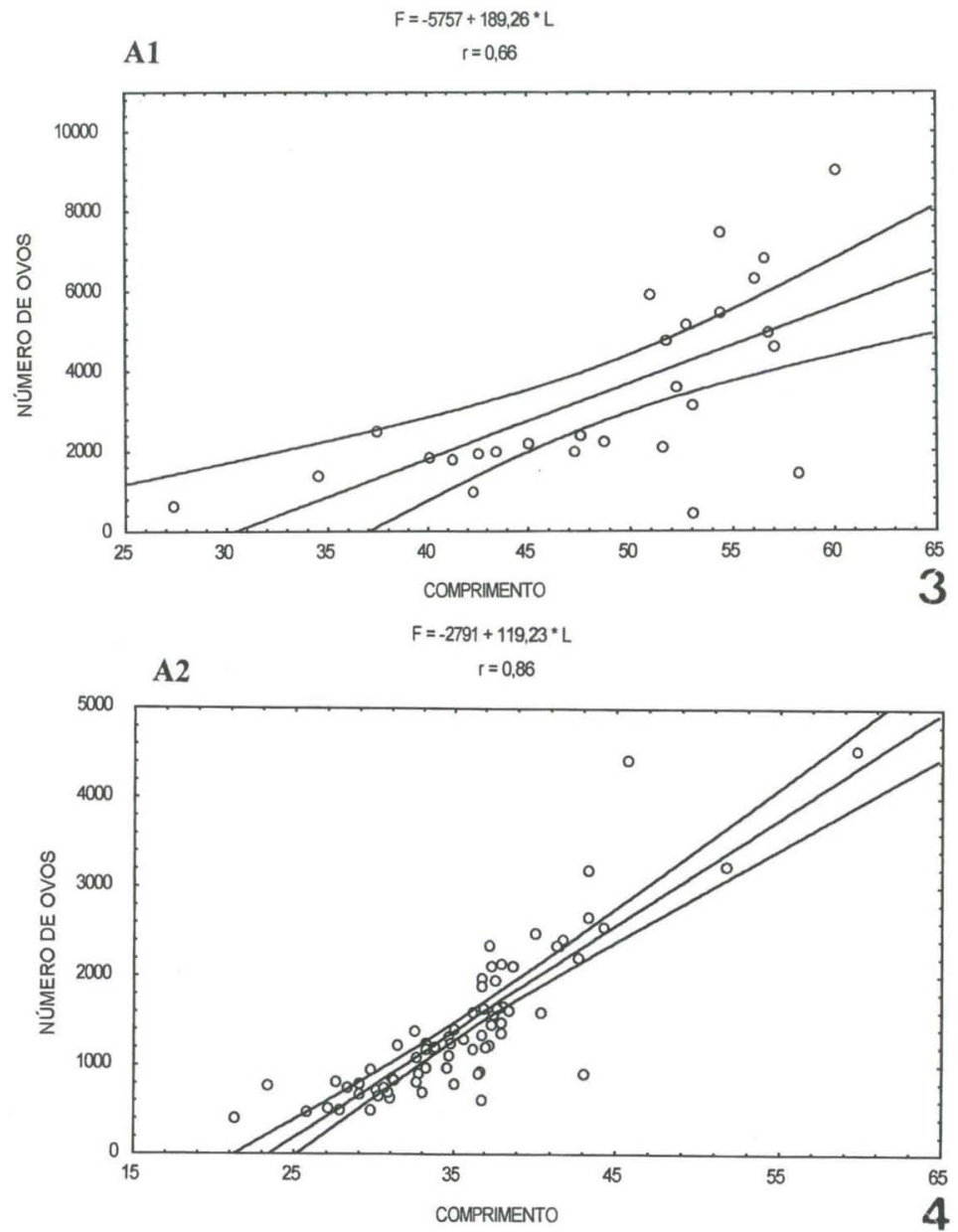

Figs 3-4. Análise de regressão linear entre o comprimento $(L)$ e o número de ovos para a estimativa da fecundidade populacional ( $F$ ) de $M$. olfersii nas áreas de estudo (3) A1 e (4) A2.

Nossos resultados demonstram que para $M$. olfersii a fecundidade é bastante distinta nas duas populações estudadas. Porém o aumento no número médio de ovos transportados pelas fêmeas, pode estar relacionado com o tamanho médio das fêmeas, confirmando a hipótese de que o porte é um dos fatores determinantes da fecundidade. Segundo OH \& HARTNOLL (1999), a fecundidade pode variar ainda de acordo com as condições fisiológicas das fêmeas, estação do ano, latitude e condições ambientais em geral. As variações na estimativa da fecundidade podem ser decorrentes de diferenças no tamanho máximo dos exemplares e das características genéticas próprias das populações de origem.

As características reprodutivas apresentadas por $M$. olfersii indicam a plasticidade da espécie em resposta às variações ambientais, objetivando seu sucesso 
reprodutivo. $\mathrm{O}$ fato de carregarem os ovos em desenvolvimento alojados na câmara incubadora, aderidos ao corpo da fêmea, até o momento da eclosão, contribui para uma maior sobrevivência de embriões, garantindo também a manutenção da espécie (SHAKUNTALA 1977).

\section{REFERÊNCIAS BIBLIOGRÁFICAS}

Adiyodi, R.G. \& T. Subramoniam. 1983. Arthropoda-Crustacea, p. 443-495. In: K.G. Adiyodi \& R.G. ADIYODI (Eds). Reproductive biology of invertebrates. New York, Wiley Press, Vol. 1, $\mathrm{XXV}+770 \mathrm{p}$.

Boolootian, R.A.; A.C. Giese; A. Farmafarmaian \& J. Tucker. 1959. Reproductive cycles of five west coast crabs. Physiol. Zool. 32 (4): 213-220.

Bond-Buckup, G. \& L. Buckup. 1989. Os Palaemonidae de águas continentais do Brasil Meridional (Crustacea, Decapoda). Rev. Brasil. Biol. 49 (4): 883-896.

BoscHI, E.E. 1974. Biologia de los crustaceos cultivables en America Latina. Carpas 06: 1-24.

Carvalho, H.A. 1978. Análise dos métodos de estudo do ciclo sexual em crustáceos (Crustacea, Natantia). Natura $78(4)$ : 67-76.

CECA/FNMA. 1996. Uma cidade numa ilha - Relatório sobre os problemas sócio-ambientais da Ilha de Santa Catarina. Florianópolis, Ed. Insular, 247p.

COREY, S. \& D.M. REID. 1991. Comparative fecundity of decapod crustaceans I. The fecundity of thirty-three species of nine families of caridean shrimp. Crustaceana 60 (3): 270-294.

Dumbauld, B.R.; D.A. Armstrong \& K.L. Feldman. 1996. Life-history characteristics of two sympatric thalassinidean shrimps, Neotrypaea californiensis and Upogebia pugettensis, with implications for oyster culture. Jour. Crust. Biol. 16 (4): 689-708.

Fingerman, M. 1995. Endocrine mechanisms in crayfish, with emphasis on reproduction and neurotransmitter regulation of hormone release. Amer. Zool. 35: 68-78.

GIESE, A.C. \& J.S. PEARSE. 1974. Reproduction of Marine Invertebrates. New York, Academic Press, Vol. 1, XI+546p.

GrazZiani, C.A.; K.S. Chung \& M. DE Donato. 1993. Comportamiento reproductivo y fertilidad de Macrobrachium carcinus (Decapoda: Palaemonidae) en Venezuela. Rev. Biol. Trop. 41 (3): 657-665.

Hartnoll, R.G. 1982. Growth, p. 111-196. In: L.G. Abele (Ed.). Embryology, Morphology and Genetics. New York, Academic Press, XXI+439p.

LAUFER, H. \& M. LANDAU. 1991. Endocrine control of reproduction in shrimp and other Crustacea, p. 65-81. In: P.F. DE LOACH; W.J. Dougherty \& M.A. Davidson (Eds). Developments in Aquaculture and Fisheries Science: frontiers of shrimp research. Amsterdam, Elsevier, Vol. 22, VII $+294 \mathrm{p}$.

LimA, A.G.; J.C. MCNAMARA; W.R. TerRA. 1997. Regulation of hemolymp osmolytes and gill $\mathrm{Na}^{+} / \mathrm{K}^{+}$ - ATPase activities during acclimation to saline media in the freshwater shrimp Macrobrachium olfersii (Wiegmann, 1836) (Decapoda, Palaemonidae). Jour. Exp. Mar. Biol. Ecol. 215: 81-91.

McNamara, J.C.; G.S. Moreira \& P. Moreira. 1985. Thermal effects on metabolism in selected shrimps Macrobrachium olfersii and Macrobrachium heterochirus (Decapoda, Palaemonidae). Comp. biochm. Physiol. 80A (2): 187-190.

MÜLLER, Y.M.R. \& A.C. PRAZERES. 1992. Influência da salinidade e temperatura da água sobre a captura de Macrobrachium olfersii (Wiegmann, 1836) coletados no canal da Lagoa do Peri -Florianópolis/SC. Acta Limnol. Brasil. 4: 175-183

Muller, Y.M.R.; E.M. NAZARI; C.M. Bressan \& D. Ammar. 1996. Aspectos da reprodução de Palaemon pandaliformis (Stimpson) (Decapoda, Palaemonidae) no manguezal de Ratones, Santa Catarina. Rev. bras. Zool. 13 (3): 633-642.

Müller, Y.M.R.; E.M. Nazari; D. Ammar; E. Cargnin-Ferreira; I.T. Beltrame \& C. Pacheco. 1999. Biologia dos Palaemonidae (Crustacea, Decapoda) da bacia hidrográfica de Ratones, Florianópolis, Santa Catarina, Brasil. Revta bras. Zool. 16 (3): 629-636.

Revta bras. Zool. 18 (2): 529 - 537, 2001 
OH, C.W. \& R.G. Hartnoll. 1999. Size at sexual maturity, reproductive output, and seasonal reproduction of Philocheras trispinosus (Decapoda) in Port Erin Bay, Isle of Man. Jour. Crust. Biol. 19 (2): 252-259.

PereirA, G.A. \& J.V. Garcia D. 1995. Larval development of Macrobrachium reyesi Pereira (Decapoda: Palaemonidae), with a discussion on the abbreviated development in palaemonids. Jour. Crust. Biol. 15 (1): 117-133.

PERrone, E.C. \& F. Vieira. 1990. Ocorrência e período reprodutivo de Eleotris pisonis (Teleostei: Eleotrididae) na região estuarina do Rio Jucu, Espírito Santo, Brasil. Ciência Cultura 42 (9): 707-710.

QUACKENBUSH, L.S. 1994. Lobster reproduction: a review. Crustaceana 67 (1): 82-94.

Shakuntala, K. 1977. The Relation Between Body Size and Number of Eggs in the Freshwater Prawn Macrobrachium lamarrei (Decapoda, Caridae). Crustaceana 33 (1): 17-21.

VAlentI, W.C.; J.T.C. MELlo \& V.L. LoBÃo. 1989. Fecundidade em Macrobrachium acanthurus (Wiegmann, 1836) do Rio Ribeira do Iguape (Crustacea, Decapoda, Palaemonidae). Revta bras. Zool. 6 (1): 9-15.

Recebido em 10.VIII.2000; aceito em 30.V.2001. 